\title{
High-shear-velocity Composite Acoustic Materials of AlN/Diamond for Biological MEMS Devices
}

\author{
Hsi-Shan Huang ${ }^{1}$ and Sean $\mathrm{Wu}^{2 *}$ \\ ${ }^{1}$ College of Robotics, Fuzhou Polytechnic, \\ No. 8 Lianrong Road, Fuzhou University Town, Fujian Province 350108, China \\ ${ }^{2}$ Department of Digital Game and Animation Design, Tung Fang Design University, \\ No. 110, Dongfang Rd., Hunei Dist., Kaohsiung City 82941, Taiwan
}

(Received July 13, 2018; accepted October 24, 2018)

Keywords: SH SAW, (100) AlN, diamond, sensors

Shear horizontal (SH) surface acoustic wave (SAW) sensors formed by combining (100) AlN films with a diamond substrate have been developed in this research. The propagation characteristics of SAW in four composite structures according to the positions of interdigital transducer (IDT) electrodes and/or thin metal films were investigated theoretically. Those composite SAW substrates exhibited excellent SH SAW properties and have the potential for further application in biological and liquid SAW sensors.

\section{Introduction}

Some special piezoelectric crystals with a special cut can excite shear horizontal ( $\mathrm{SH}$ ) surface acoustic waves (SAWs), which are very good for liquid and biological SAW sensors. ${ }^{(1-10)}$ SAW sensors are based on the mass loading effect. Therefore, a high SAW velocity will make SAW sensors work at a high frequency to have a high sensitivity. Therefore, a high-velocity SH SAW is important for the development of high-sensitivity liquid and biological SAW sensors.

AlN films have been widely investigated for application in SAW and film bulk acoustic wave (FBAW) devices because of their high SAW velocity and suitable piezoelectric coupling factor. Diamond is an attractive non-piezoelectric material for high-velocity SAW devices because it has the highest SAW velocity among all materials and needs to add a piezoelectric layer on the top to excite SAWs. There are many researchers studying the Rayleigh SAW of (002) AlN films on (111) diamond for application in high-velocity SAW substrates. ${ }^{(11-20)}$ Different orientations of piezoelectric films will form different acoustic properties. Recently, the Rayleigh SAW mode of (100) AlN films on (111) diamond with propagation along the $z$-axis has been studied and excellent SAW properties were exhibited. ${ }^{(21-23)}$

Hexagonal crystalline substrates with the $z$-axis parallel to the surface have a pure $\mathrm{SH}$ SAW propagating along the direction perpendicular to the $z$-axis. ${ }^{(24-27)}$ AlN films are also hexagonal crystalline structures. In our previous research, (100) AlN films on (111) diamond

*Corresponding author: e-mail: wusean.tw@gmail.com https://doi.org/10.18494/SAM.2019.2111 
with propagation along the $y$-axis can excite high-velocity SH SAWs. ${ }^{(28)}$ For a composite thinfilm SH SAW substrate, there are four basic structures, namely, interdigital transducer (IDT)/ (100)AlN/(111)diamond, (100)AlN/IDT/(111)diamond, IDT/(100)AlN/metal/(111)diamond, and metal/IDT/(100)AlN/(111)diamond. Different composite structures will form different SH SAW properties. The first five SH SAW modes in the four-layered structures with propagation along the $y$-axis will be theoretically analyzed in this research.

\section{Method of Analysis}

Following an approach similar to that developed by Campbell and Jones, ${ }^{(29)}$ the matrix method is effectively employed here to calculate the SH SAW velocity in a layered piezoelectric structure.

The acoustic and electric fields in mediums 1 and 2 can be expressed as

$$
\begin{gathered}
u_{j}^{(1)}=\sum_{m=1}^{4} C_{m} a_{j}^{(m)} \exp \left(i k b^{(m)} z\right) \exp [i k(P x-v t)], j=1,2,3, \\
\phi^{(1)}=\sum_{m=1}^{4} C_{m} a_{4}^{(m)} \exp \left(i k b^{(m)} z\right) \exp [i k(P x-v t)], \\
u_{j}^{(2)}=\sum_{m=1}^{8} X_{m} \alpha_{j}^{(m)} \exp \left(i k \beta^{(m)} z\right) \exp [i k(P x-v t)], \\
u_{j}^{(2)}=\sum_{m=1}^{8} X_{m} \alpha_{4}^{(m)} \exp \left(i k \beta^{(m)} z\right) \exp [i k(P x-v t)],
\end{gathered}
$$

where $u$ is the acoustic displacement, $\phi$ the electric potential, $v$ the phase velocity, $k$ the wave number in the $x$-direction, $P=1+i \gamma, \gamma$ the attenuation coefficient, $\beta$ the wave number ratios, and $\alpha$ the associated partial field amplitude. Substituting Eqs. (1) and (2) into stiffened Christoffel equations yields an eight-order algebraic equation for the wave number ratio $b$. Thus, for each pair of $(v, \gamma)$ values, there are eight real or complex $b$ values. For a semi-infinite piezoelectric crystal, i.e., medium 1 in this structure, four complex roots with negative imaginary parts are selected for the Rayleigh type. In medium 2 , all eight roots of $\beta$ are selected.

The boundary conditions require that the acoustic displacements and stresses should be continuous at $d=0$, and that the stress-free surface is $d=h$. In addition, the electric potential and normal component of electric displacement must be continuous at the interface for an electrically free surface. For a metalized (thin metal film) surface, the electric potential is not observed. By substituting Eqs. (1)-(4) into the boundary conditions, the phase velocity $v$ and the attenuation coefficient $\gamma$ can be obtained numerically. The electromechanical coupling coefficient $\left(K^{2}\right)$ can be calculated from 


$$
K^{2}=2 \frac{v_{f}-v_{m}}{v_{f}}
$$

where $v_{f}$ and $v_{m}$ are the phase velocities obtained when the electrical boundary conditions at the interface at which the IDT is placed are assumed to be electrically free and shorted, respectively. For material constants, refer to Refs. 19 and 30.

\section{Results and Discussion}

The phase velocity dispersion curves of SH SAW mode propagation in the IDT/(100)AIN/(111) diamond structure are shown in Fig. 1. The curves are plotted as functions of the film thickness ratio $(h / \lambda)$, where $h$ is the AlN film thickness and $\lambda$ is the wavelength. The phase velocity of each mode decreases as the film thickness ratio increases. Modes $0,1,2,3$, and 4 show cutoff at the critical point, where the phase velocity is equal to the shear bulk wave velocity in diamond $(12323 \mathrm{~m} / \mathrm{s})$. Mode 1 occurs at $h / \lambda>0.37$, mode 2 occurs at $h / \lambda>0.64$, mode 3 occurs at $h / \lambda>0.91$, and mode 4 occurs at $h / \lambda>1.12$. As $h / \lambda$ increases, the phase velocity curve decreases. The $K^{2}$ dispersion curves of the first five SH SAW modes propagating in the four composite structures are shown in Figs. 2-5. As regards the IDT/(100)AIN/(111)diamond structure in Fig. 2, the curves become smoother and smaller as the first five SH SAW modes increase. For mode 0, the $K^{2}$ curve shows a maximum value $(1.27 \%)$ at $h / \lambda=0.28$. For mode 1 , the $K^{2}$ curve shows a maximum value $(0.46 \%)$ at $h / \lambda=0.7$. For mode 2 , the $K^{2}$ curve shows a maximum value $(0.27 \%)$ at $h / \lambda=1.21$. For mode 3 , the $K^{2}$ curve shows a maximum value $(0.196 \%)$ at $h / \lambda=1.7$. For mode 4 , the $K^{2}$ curve shows a maximum value $(0.153 \%)$ at $h / \lambda=2.19$. As regards the (100)AlN/IDT/

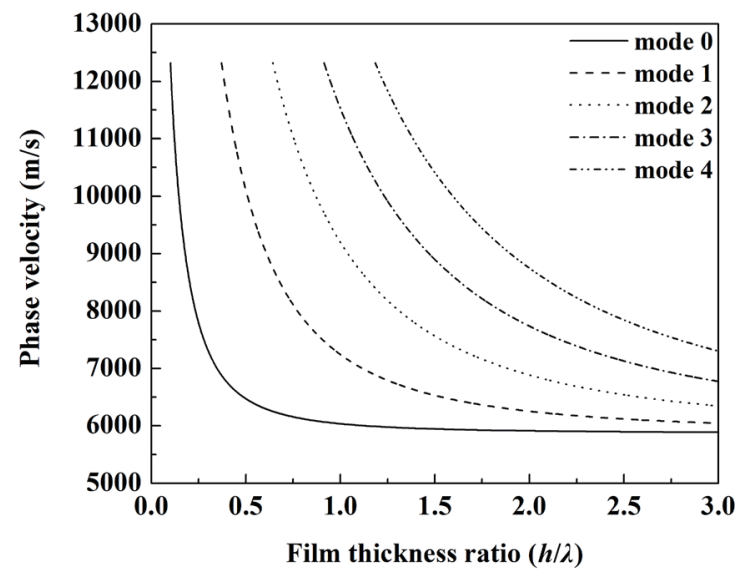

Fig. 1. Calculated phase velocity dispersion curves of first five SH SAW modes propagating in (100)AlN/ (111)diamond structure.

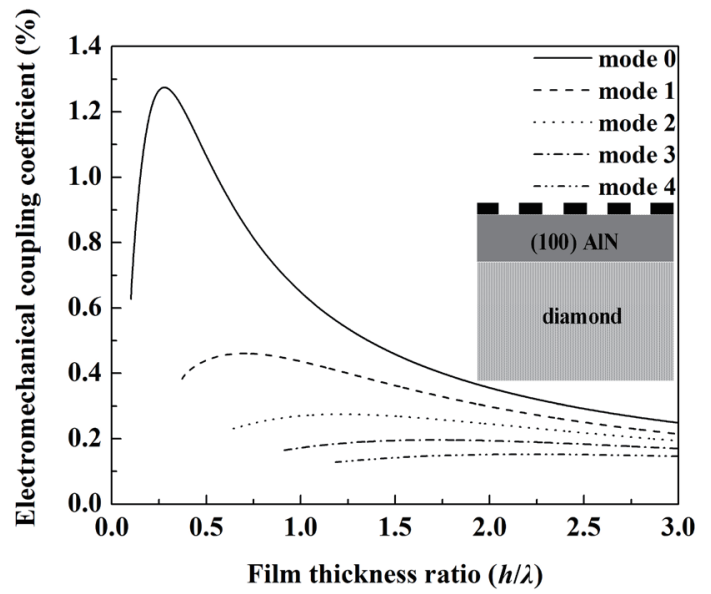

Fig. 2. Calculated $K^{2}$ dispersion curves of first five SH SAW modes propagating in IDT/(100)AIN/(111) diamond. 
(111)diamond structure in Fig. 3, the $K^{2}$ of each mode decreases rapidly as the film thickness ratio increases and the maximum $K^{2}$ occurs at the critical point. For mode 0 , the maximum $K^{2}$ is $26.82 \%$. For mode 1 , the maximum $K^{2}$ is $14.38 \%$. For mode 2 , the maximum $K^{2}$ is $10.31 \%$. For mode 3 , the maximum $K^{2}$ is $8.17 \%$. For mode 4 , the maximum $K^{2}$ is $6.82 \%$. As regards the IDT/(100)AlN/metal/(111)diamond structure in Fig. 4, the curves become smoother and smaller as the first five SH SAW modes increase. For mode 0 , the $K^{2}$ curve shows a maximum value (1.2\%) at $\mathrm{h} / \lambda=0.29$. For mode 1 , the $K^{2}$ curve shows a maximum value $(0.46 \%)$ at $h / \lambda=0.7$. For mode 2 , the $K^{2}$ curve shows a maximum value $(0.27 \%)$ at $h / \lambda=1.26$. For mode 3 , the $K^{2}$ curve shows a maximum value $(0.19 \%)$ at $h / \lambda=1.8$. For mode 4 , the $K^{2}$ curve shows a maximum value $(0.15 \%)$ at $h / \lambda=2.3$. As regards the metal/(100)AlN/IDT/(111)diamond structure in Fig. 5, the $K^{2}$ of

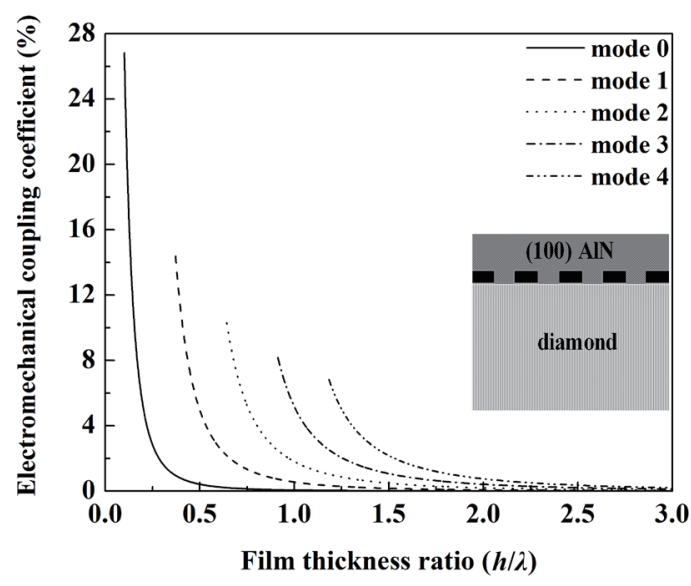

Fig. 3. Calculated $K^{2}$ dispersion curves of first five SH SAW modes propagating in (100)AlN/IDT/(111) diamond.

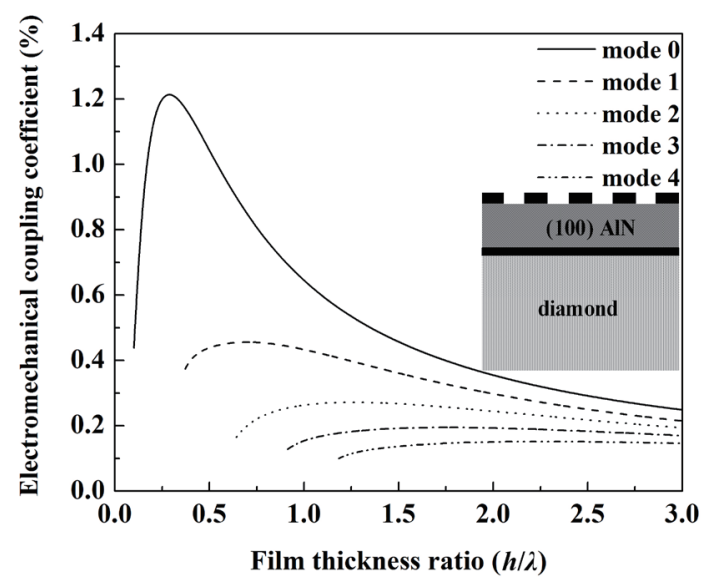

Fig. 4. Calculated $K^{2}$ dispersion curves of first five SH SAW modes propagating in IDT/(100)AlN/metal/ (111)diamond.

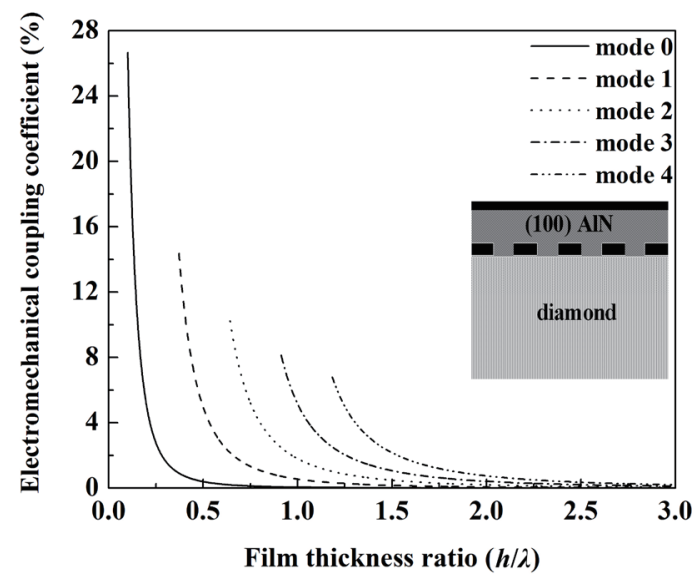

Fig. 5. Calculated $K^{2}$ dispersion curves of first five SH SAW modes propagating in metal/(100)AlN/IDT/(111) diamond. 
Table 1

Relative maximum $K^{2}$ values of the first five modes propagating in the four structures.

\begin{tabular}{|c|c|c|c|c|}
\hline Propagation mode & Structure & $h / \lambda$ & $K^{2}(\%)$ & Velocity $(\mathrm{m} / \mathrm{s})$ \\
\hline \multirow{4}{*}{ Mode 0} & IDT/(100)AlN/diamond & 0.28 & 1.27 & 7430 \\
\hline & (100)AlN/IDT/diamond & 0.1 & 26.82 & 10669 \\
\hline & IDT/(100)AlN/metal/diamond & 0.29 & 1.2 & 7275 \\
\hline & metal/(100)AlN/IDT/diamond & 0.1 & 26.66 & 10645 \\
\hline \multirow{4}{*}{ Mode 1} & IDT/(100)AlN/diamond & 0.7 & 0.46 & 8357 \\
\hline & (100)AlN/IDT/diamond & 0.37 & 14.38 & 11436 \\
\hline & IDT/(100)AlN/metal/diamond & 0.7 & 0.46 & 8288 \\
\hline & metal/(100)AlN/IDT/diamond & 0.37 & 14.37 & 11415 \\
\hline \multirow{4}{*}{ Mode 2} & IDT/(100)AlN/diamond & 1.21 & 0.27 & 8299 \\
\hline & (100)AlN/IDT/diamond & 0.64 & 10.31 & 11687 \\
\hline & IDT/(100)AlN/metal/diamond & 1.26 & 0.27 & 8106 \\
\hline & metal/(100)AlN/IDT/diamond & 0.64 & 10.25 & 11677 \\
\hline \multirow{4}{*}{ Mode 3} & IDT/(100)AlN/diamond & 1.7 & 0.196 & 8322 \\
\hline & (100)AlN/IDT/diamond & 0.91 & 8.17 & 11818 \\
\hline & IDT/(100)AlN/metal/diamond & 1.8 & 0.19 & 8073 \\
\hline & metal/(100)AlN/IDT/diamond & 0.91 & 8.13 & 11810 \\
\hline \multirow{4}{*}{ Mode 4} & IDT/(100)AlN/diamond & 2.19 & 0.153 & 8335 \\
\hline & (100)AlN/IDT/diamond & 1.12 & 6.82 & 11901 \\
\hline & IDT/(100)AlN/metal/diamond & 2.3 & 0.15 & 8120 \\
\hline & metal/(100)AlN/IDT/diamond & 1.12 & 6.79 & 11895 \\
\hline
\end{tabular}

each mode decreases rapidly as the film thickness ratio increases and the maximum $K^{2}$ occurs at the critical point. For mode 0, the maximum $K^{2}$ is $26.66 \%$. For mode 1 , the maximum $K^{2}$ is $14.37 \%$. For mode 2, the maximum $K^{2}$ is $10.25 \%$. For mode 3, the maximum $K^{2}$ is $8.13 \%$. For mode 4 , the maximum $K^{2}$ is $6.79 \%$.

For the first five modes of the four structures, the relative maximum $K^{2}$ values are summarized in Table 1. It is obvious that mode 1 of the (100)AlN/IDT/diamond structure has a maximum $K^{2}(26.82 \%)$ and a minimum film thickness ratio $(0.1)$, where the velocity is $10669 \mathrm{~m} / \mathrm{s}$. Mode 4 of the (100)AIN/IDT/diamond structure has a maximum velocity $(11901 \mathrm{~m} / \mathrm{s})$ at $h / \lambda=1.12$, where $K^{2}$ is $6.82 \%$.

\section{Conclusions}

In this research, (100) AlN films were combined with diamond to form high-velocity SH SAW substrates including four composite structures, namely, IDT/(100)AlN/(111)diamond, (100)AlN/IDT/(111)diamond, IDT/(100)AlN/metal/(111)diamond, and metal/(100)AlN/IDT/(111) diamond. The structures exhibited excellent SH SAW properties. The research results provide a predictable theoretical basis for the further application of such substrates in SH SAW devices.

\section{Acknowledgments}

We thank Fuzhou Polytechnics for financial support (project code: RCQD201701). 


\section{References}

1 S. T. Ten, U. Hashim, S. C. B. Gopinath, W. W. Liu, K. L. Foo, S. T. Sam, S. F. A. Rahman, C. H. Voon, and A. N. Nordin: Biosens. Bioelectron. 93 (2017) 146. https://doi.org/10.1016/j.bios.2016.09.035

2 K. Tada, T. Nozawa, and J. Kondoh: Jpn. J. Appl. Phys. 56 (2017) 07JD15. http://iopscience.iop.org/ article/10.7567/JJAP.56.07JD15/meta

3 J. Luo, A. Quan, C. Fu, and H. Li: J. Alloys Compd. 693 (2017) 558. https://doi.org/10.1016/j.jallcom.2016.09.118

4 C. Fu, A. J. Quan, J. T. Luo, H. F. Pang, Y. J. Guo, Q. Wu, W. P. Ng, X. T. Zu, and Y. Q. Fu: Appl. Phys. Lett. 110 (2017) 173501. https://doi.org/10.1063/1.4982073

5 B. Zhang, T. Han, G. Tang, Q. Zhang, T. Omori, and K. Hashimoto: Jpn. J. Appl. Phys. 56 (2017) 07 JD02. http://iopscience.iop.org/article/10.7567/JJAP.56.07JD02/meta

6 A. Vikström and M. V. Voinova: Sens. Bio-Sens. Res. 11 (2016) 78. https://doi.org/10.1016/j.sbsr.2016.08.004

7 T. M. A. Gronewold: Anal. Chim. Acta 603 (2007) 119. https://doi.org/10.1016/j.aca.2007.09.056

8 M. Bisoffi, B.Hjelle, D. C. Brown, D. W. Branch, T. L. Edwards, S. M. Brozik, V. S. Bondu-Hawkins, and R. S. Larson: Biosens. Bioelectron. 23 (2008) 1397. https://doi.org/10.1016/j.bios.2007.12.016

9 F. Martin, M. I. Newton, G. McHale, K. A. Melzak, and E. Gizeli: Biosens. Bioelectron. 19 (2004) 627. https:// doi.org/10.1016/S0956-5663(03)00257-4

10 F. Herrmann, M. Weihnacht, and S. Biittgenbach: IEEE Trans. Ultrason. Ferroelectr. Freq. Control 48 (2001) 268. https://doi.org/10.1109/58.896139

11 L. Chen, H. Liu, S. Liu, C. Li, Y. Wang, K. An, C. Hua, J. Liu, J. Wei, L. Hei, and F. Lv: Appl. Surf. Sci. 431 (2018) 152. https://doi.org/10.1016/j.apsusc.2017.09.036

12 M. Sznajder and R. Hrytsak: Diam. Relat. Mater. 83 (2018) 94. https://doi.org/10.1016/j.diamond.2018.01.027

13 L. Wang, S. Chen, J. Zhang, D. Xiao, K. Han, X. Ning, J. Liu, Z. Chen, and J. Zhou: Appl. Phys. Lett. 111 (2017) 253502. https://doi.org/10.1063/1.5006884

14 C. Caliendo and M. Hamidullah: Proc. 4th Int. Electronic Conf. Sensors and Applications 2 (2017) 133. https:// doi.org/10.3390/ecsa-4-04924

15 L. Wang, S. M. Chen, X. Ning, Z. Chen, J. T. Liu, and J. Y. Zhang: Joint IEEE Int. Symp. Applications of Ferroelectric (ISAF) (IEEE, 2017). https://doi.org/10.1109/ISAF.2017.8000223

16 J. G. Rodriguez-Madrid, G. F. Iriarte, J. Pedros, O. A. Williams, D. Brink, and F. Calle: IEEE Electr. Device Lett. 33 (2012) 495. https://doi.org/10.1109/LED.2012.2183851

17 V. Mortet, O. Elmazria, M. Nesladek, M. B. Assouar, G. Vanhoyland, J. D’Haen, M. D’Olieslaeger, and P. Alnot: Appl. Phys. Lett. 81 (2002) 1720. https://doi.org/10.1063/1.1503875

18 G. F. Iriarte: J. Appl. Phys. 93 (2003) 9604. https://doi.org/10.1063/1.1574172

19 M. Benetti, D. Cannata, F. D. Pietrantonio, and E. Verona: IEEE Trans. Ultrason. Ferroelectr. Freq. Control 52 (2005) 1806. https://doi.org/10.1109/TUFFC.2005.1561635

20 P. Kirsch, M. B. Assouar, O. Elmazria, V. Mortet, and P. Alnot: Appl. Phys. Lett. 88 (2006) 223504. https:// doi.org/10.1063/1.2208372

21 R. Ro, Y. F. Chiang, C. C. Sung, R. Lee, and S. Wu: IEEE Trans. Ultrason. Ferroelectr. Freq. Control 57 (2010) 46. https://doi.org/10.1109/FREQ.2009.5168219

22 S. Wu, R. Ro, Z. X. Lin, and M. S. Lee: J. Appl. Phys. 104 (2008) 064919. https://doi.org/10.1063/1.2986215

23 Z. X. Lin, S. Wu, R. Ro, and M. S. Lee: IEEE Trans. Ultrason. Ferroelectr. Freq. Control 56 (2009) 1246. https://doi.org/10.1109/TUFFC.2009.1166

24 J. L. Bleustein: Appl. Phys. Lett. 13 (2003) 412. https://doi.org/10.1063/1.1652495

25 Y. V. Gulyaev: Soviet Phys. JETP Lett. 9 (1969) 37.

26 K. Nakamura, T. Shoji, and H. B. Kang: Jpn. J. Appl. Phys. 39 (2000) L534. http://iopscience.iop.org/ article/10.1143/JJAP.39.L534

27 F. S. Hickernell: IEEE Trans. Ultrason. Ferroelectr. Freq. Control 52 (2005) 809. https://doi.org/10.1109/ TUFFC.2005.1503966

28 S. Wu, R. Ro, Z. X. Lin, and M. S. Lee: Appl. Phys. Lett. 94 (2009) 092903. https://doi.org/10.1063/1.3093528

29 J. J. Campbell and W. R. Jones: IEEE Trans. Sonics and Ultrason. 15 (1968) 209. https://doi.org/10.1109/ T-SU.1968.29477

30 J. G. Gualtieri, J. A. Kosinski, and A. Ballato: IEEE Trans. Ultrason. Ferroelectr. Freq. Control 41 (1994) 53. https://doi.org/10.1109/58.265820 


\section{About the Authors}

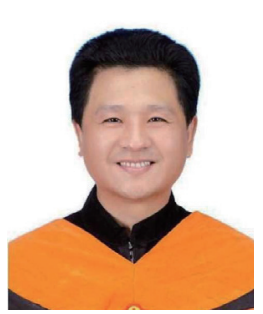

Hsi-Shan Huang is currently a professor and the dean of the College of Robotics at Fuzhou Polytechnic, Fujian Province, China. His areas of interest include aviation electronic engineering, renewable energy technology engineering, and fiber optic component guidance theory. He received his M.S. degree in information management engineering from the University of Costa Rica in 2004 and Ph.D. degree in photoelectric engineering from Kaohsiung First University of Science and technology in 2009. (hw5400@hotmail.com)

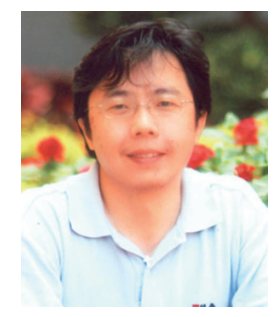

Sean Wu was born in Kaohsiung, Taiwan, on December 30, 1968 . He received his B.S. degree in electrical engineering in 1993 from Chung Yuan Christian University in Taiwan. He received his M.S. and Ph.D. degrees in electrical engineering from National Cheng Kung University in 1995 and 2001, respectively. He joined the Department of Electronics Engineering and Computer Science, Tung Fang Design University and became a professor in 2010. He also served as the Department Head of Electronics Engineering and Computer Science from 2006 to 2008. He was the Dean of Academic Affairs of Tung Fang Design University from 2011 to 2013. He was the recipient of the 2012 special talent excellent elasticity professor salary of the Taiwan Ministry of Education annual awards. His research interests are in the fabrication of piezoelectric thin films, design of acoustic wave devices, and substrate materials for SAW and FBAR devices. He has authored or coauthored over 100 technical journal and conference papers.

(wusean.tw@gmail.com) 